\title{
Photocatalytic degradation of methylene blue under visible light using PVP-capped ZnS and CdS nanoparticles
}

\begin{abstract}
Photocatalysis based on semiconductor quantum dots which utilize the solar energy can be used for the elimination of pollutants from aqueous media and applied for water purification. Degradation of dyes is a standard method to check the photocatalytic activity of any type of photocatalyst. In this paper polyvinyl pyrrolidone (PVP)-capped ZnS and CdS nanoparticles were prepared by a simple microwave irradiation method and studied in detail for their photocatalytic activity in visible range. The obtained nanoparticles were characterized by XRD, TEM, UVÏ Vis and EDX. The prepared PVP-capped ZnS and CdS nanoparticles have average sizes of $\sim 5.1$ and $18.3 \mathrm{~nm}$ with cubic and hexagonal crystalline structures, respectively. PVP capped CdS nanoparticles exhibited a unique property of optical absorption in visible region with a wave length below than $460 \mathrm{~nm}$ followed by a clear long tail up to $700 \mathrm{~nm}$ and showed excellent activity toward degradation of dye under visible light illumination. The photocatalytic activity of PVP-capped CdS nanoparticles was found to be improved by mixing with appropriate amount of PVP-capped $\mathrm{ZnS}$ nanoprticles. From the study of variation in weight percentages of PVP-capped $\mathrm{ZnS}$ nanoparticles, the physical mixture with $20 \%$ of PVP-capped $\mathrm{ZnS}$ nanoparticles was found to be very efficient for degradation of methylene blue. In this case the degradation efficiency after $6 \mathrm{~h}$ illumination was about $81 \%$.
\end{abstract}

Keyword: PVP; ZnS and CdS nanoparticles; Photocatalysis; Visible light 